\title{
An EPR Investigation of Binding Environments by N-Donor Chelating Exchange Resins for Cu Extraction from Aqueous Media
}

\author{
Jacob Spencer, ${ }^{\dagger}$ James Stevens, $^{\dagger}$ Christopher Perry, ${ }^{\S}$ and Damien M. Murphy ${ }^{*}, \dagger \odot$ \\ ${ }^{\dagger}$ School of Chemistry, Cardiff University, Park Place, Cardiff CF10 3AT, United Kingdom \\ "Johnson Matthey Technology Center, Blounts Court Road, Sonning Common, Reading RG4 9NH, United Kingdom

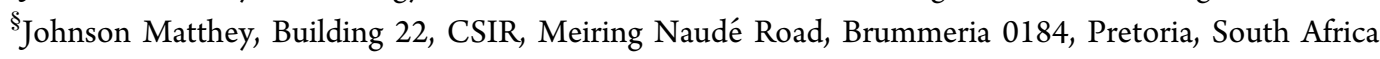

Supporting Information

ABSTRACT: Chelating exchange resins (CERs) are now widely used for metal extraction in aqueous acidic media. Many of these CERs contain surface $\mathrm{N}$-donor ligands, such as di(2-picolylamine) (BPA) and picolylamine (PA), which are highly selective for $\mathrm{Cu}$ (II) uptake. Two such widely used resins are Dowex M4195 and CuWRAM. Surprisingly, very little is known about the $\mathrm{Cu}(\mathrm{II})$ binding environments on the exchange resins, particularly at variable concentrations and $\mathrm{pH}$ 's, and therefore we used EPR spectroscopy to investigate this binding. The broad EPR spectra of the $\mathrm{Cu}(\mathrm{II})$ loaded resins are quite complex, indicating the presence of multiple $\mathrm{Cu}(\mathrm{II})$ binding environments.
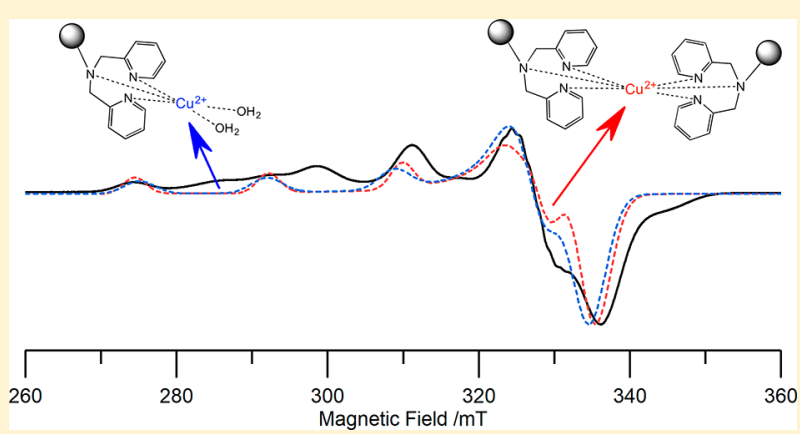
By preparing a series of well-defined $\left[\mathrm{Cu}^{\mathrm{II}}(\mathrm{PA})_{x}\right]$ and $\left[\mathrm{Cu}^{\mathrm{II}}(\mathrm{BPA})_{x}\right]$ complexes and studying their EPR and UV-vis spectra, the individual $\mathrm{Cu}(\mathrm{II})$ species contributing to the broad and overlapping EPR spectra of the loaded resins were identified. For Dowex M4195, $\left[\mathrm{Cu}^{\mathrm{II}}(\mathrm{BPA})\right]\left(\mathrm{H}_{2} \mathrm{O}\right)_{m}$ and $\left[\mathrm{Cu}^{\mathrm{II}}(\mathrm{BPA})_{2}\right]$ complexes are most dominant, whereas for CuWRAM two dominant species including $\left[\mathrm{Cu}^{\mathrm{II}}(\mathrm{PA})_{2}\right]\left(\mathrm{H}_{2} \mathrm{O}\right)_{m}$ and $\left[\mathrm{Cu}^{\mathrm{II}}(\mathrm{PA})_{3}\right]$ were identified. Notably, $\left[\mathrm{Cu}^{\mathrm{II}}(\mathrm{PA})\right]\left(\mathrm{H}_{2} \mathrm{O}\right)_{m}$ was not present in this sample. The experimental spin Hamiltonian parameters for all these species were in good agreement with the density functional theory derived values. Additional intermolecular $\mathrm{Cu}(\mathrm{II})$ species were identified on both resins, labeled $\left[\mathrm{Cu}^{\mathrm{II}}(\mathrm{BPA})_{x}(\mathrm{BPA})_{y}\left(\mathrm{H}_{2} \mathrm{O}\right)_{n}\right]$ and $\left[\mathrm{Cu}^{\mathrm{II}}(\mathrm{PA})_{x}(\mathrm{PA})_{y}\left(\mathrm{H}_{2} \mathrm{O}\right)_{n}\right]$. The presence of coordinated water in these intermolecular anchored sites was confirmed in a series of dehydration-rehydration experiments. Furthermore, a series of acid elution experiments also confirmed that these species are less strongly coordinated to the resins compared to the intramolecular species $\left[\mathrm{Cu}{ }^{\mathrm{II}}(\mathrm{BPA})\right]\left(\mathrm{H}_{2} \mathrm{O}\right)_{m},\left[\mathrm{Cu} \mathrm{u}^{\mathrm{II}}(\mathrm{BPA})_{2}\right]$, $\left[\mathrm{Cu}^{\mathrm{II}}(\mathrm{PA})_{2}\right]\left(\mathrm{H}_{2} \mathrm{O}\right)_{m}$, and $\left[\mathrm{Cu}^{\mathrm{II}}(\mathrm{PA})_{3}\right]$. Finally, while equilibrium batch uptake measurements revealed that the CuWRAM material had a much lower $\mathrm{Cu}$ (II) capacity compared to the polymeric Dowex material, the adsorbed copper can be recovered more efficiently using acid elution.

\section{INTRODUCTION}

Worldwide, over 25 million tonnes of copper are used in various manufacturing practices, of which approximately onethird of global demand is met by recycling. A number of conventional methods for metal recovery are currently employed industrially, including chemical precipitation, ${ }^{2}$ cementation, ${ }^{3}$ solvent extraction, ${ }^{4}$ and ion exchange. ${ }^{5-7}$ While ion exchange resins (IERs) are among the most expensive technologies, these adsorbents are also among the most efficient and selective methods. Sources of copper waste, such as metallurgy processes and electroplating, are typically highly acidic and frequently contain various toxic organic byproducts and complexing agents. This presents a challenge in terms of conventional extraction methods, where additional steps may be required to neutralize or treat the effluent in order to retrieve the metal in appreciable quantities. ${ }^{8}$

Instead, it is possible that complexing agents may be introduced as active site functionalities onto resin framework supports. These specialized frameworks are known as "chelating exchange resins" (CERs), and they can be advantageously employed in metal preconcentration or recycling processes on an industrial scale. ${ }^{9-11}$ CERs are usually functionalized porous materials that coordinate to metals via a combination of ionic, electrostatic, and primarily coordinative interactions, in contrast to the active exchange of labile functional groups (as in IERs). CERs act to remove metal ions through conventional Lewis acid-base interactions between the metal substrate and immobilized ligand. Lewis base sites, in IERs, often struggle to achieve the required rates and capacities in acidic media, due to competitive protonation which reduce the site nucleophilicity. CERs containing weakly basic sites have been reported to work in acidic media, even down to $\mathrm{pH}=1$, as a sufficient number of sites remain deprotonated. Typically, CERs facilitate increased adsorption rates, efficiencies, and surface areas when compared to IERs.

Received: June 1, 2018

Published: August 16, 2018 
Metal uptake kinetics, capacities, and other operational properties are highly dependent on the choice of polymer, or support, particle sizes, and functional group attached to the polymer backbone. As such, CERs may facilitate increased adsorption rates, tuneability, and surface areas in comparison to IERs.

In this study, we have investigated the exchange properties of two typical types of commercially available CER resins, namely Dowex M4195 and CuWRAM (see Scheme 1). Both

Scheme 1. General Structures for the Two Cu(II) Exchange Resins Investigated in This Work
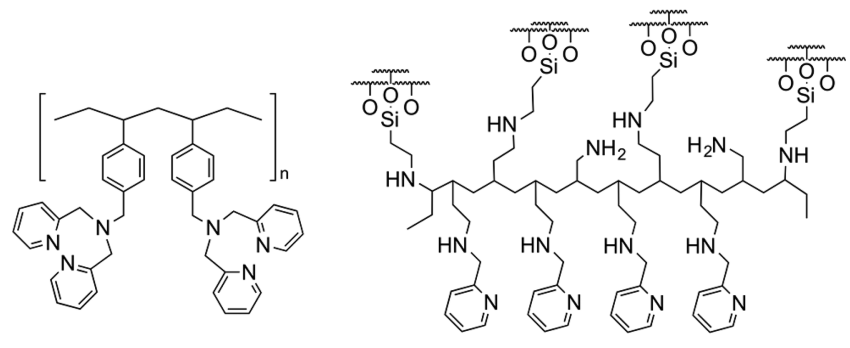

Dowex M4195

di(2-picolylamine)

CuWRAM

2-picolylamine

of these CERs employ similar N-donor ligands for the metal uptake and both have been shown to be highly selective toward $\mathrm{Cu}$ (II) uptake, even in mixed metal effluent systems. However, understanding the nature of the Lewis acid-base interaction, and indeed the $\mathrm{Cu}$ (II) binding environments which afford this high selectivity, is not straightforward.

In brief, Dowex M4195 (Scheme 1) is a macroporous, crosslinked DVB-polystyrene composite, functionalized with partially protonated tridentate $\mathrm{di}(2$-picolylamine $)$ chelating units. ${ }^{5}$ The cross-linked polymer support affords good mechanical durability, while the weakly basic di(2-picolylamine) group allows good sorption efficiency, even at low $\mathrm{pH}$ values $(\mathrm{pH}=$ 1). This is due to the presence of pyridyl nitrogen-donor atoms, which due to the electron-withdrawing effect of the heterocyclic rings, remain deprotonated even at extremely low $\mathrm{pH}$. CuWRAM on the other hand (Scheme 1) is a silicapolyamine composite bearing 2-picolylamine, developed in order to address some of the limitations of other analogous functional materials. For example, the Dowex M4195 system suffers from poor elution characteristics, with relatively strong $\mathrm{NH}_{4} \mathrm{OH}$ solutions necessary to strip most of the metal ions from the support. Whereas, the selectivity of CuWRAM is observed to be sufficient such that separation of $\mathrm{Cu}(\mathrm{II})$ is feasible even in a 250 -fold excess of $\mathrm{Zn}$ (II), ${ }^{12}$ and yet the metal is more easily eluted. Other advantages include a decreased shrink-swell action, faster operational flow rate at higher capacities, longer useable lifetimes, higher operational temperatures, and "inert" operation across a wide range of acidic and basic media. $^{13}$

While much of the past investigations on these materials have focused on the support structure, absorption properties, and uptake kinetics, ${ }^{12,14-16}$ very little is known about the local metal binding environments within CERs. For example, the nature of how the $\mathrm{Cu}$ (II) ions are actually coordinated by the supports is not clear. Many aspects of the structure, steric hindrance, and competitive equilibria, arising from the grafting of the chelate units onto a polymer, remain inadequately

described. Electron paramagnetic resonance (EPR) spectroscopy is the ideal technique to probe the local binding of copper ions by the $\mathrm{N}$-donor chelating ligands for metal extraction ${ }^{17}$ and obtain this important information. The purpose of this investigation is therefore to primarily provide a meaningful evaluation of local metal binding environments and $\mathrm{Cu}$ speciation within these two CERs, in order to address the currently unknown features associated with uptake behavior and copper coordination environments. Complementary density functional theory (DFT) and UV-vis titration data will also be reported here.

\section{EXPERIMENTAL SECTION}

Dowex M4195, $\mathrm{CuSO}_{4}$, and the free ligands 2-picolylamine (PA) and di(2-picolylamine) (BPA) were sourced from Sigma-Aldrich. Silica gel, glycerol, $\mathrm{H}_{2} \mathrm{SO}_{4}$, and $\mathrm{NH}_{4} \mathrm{OH}$ (Reagent grade) were also purchased from Sigma-Aldrich and used without further purification. CuWRAM was supplied by Johnson Matthey.

Loading Procedure. The copper loading procedure was performed on the resins Dowex M4195 and CuWRAM after an initial wash with water, followed by drying overnight in an oven at 70 ${ }^{\circ} \mathrm{C}$. A solution of $\mathrm{CuSO}_{4}$ in water, of appropriate concentration for metal loadings ( $25 \mathrm{ppm}, 40 \mathrm{~mL} / \mathrm{g}$ (resin) for $1 \mathrm{mg} / \mathrm{g}$ loading), was prepared and placed in a centrifuge tube with the appropriate mass of resin material. The slurry was mixed overnight, using a roller mixer, before collection under reduced pressure. The solid was rinsed with copious amounts of water to yield the loaded, hydrated CuWRAM and Dowex samples. Excess water was removed on filter paper, and the samples were used, without further treatment, for EPR measurements. Accurate copper contents were measured by digestion analysis and were determined to be within $10 \%$ of the target loading.

Preparation of Model Complexes. Stock solutions of $\mathrm{CuSO}_{4}$ $(80 \mathrm{mM})$ and the free ligands 2-picolylamine (hereafter abbreviated as PA) and di(2-picolylamine) (hereafter abbreviated as BPA) (4 M) were prepared in water. One $\mathrm{mL}$ samples of the $\mathrm{Cu}: \mathrm{PA}$ and $\mathrm{Cu}: \mathrm{BPA}$ complexes were prepared by mixing the $80 \mathrm{mM} \mathrm{CuSO}_{4}$ solution $(0.25$ $\mathrm{mL}$ ), with the appropriate volume of $4 \mathrm{M} \mathrm{PA}$ or BPA ligand solutions and diluted with water to achieve the desired molar ratios $(\mathrm{Cu}: \mathrm{L} ; \mathrm{L}=$ PA, BPA) 1:0.1, 1:0.5, 1:1, 1:2, 1:5, 1:10, and 1:50. The volume was made up with water and glycerol $(40 \% \mathrm{v} / \mathrm{v})$ to achieve the desired concentration $\left(20 \mathrm{mM} \mathrm{CuSO}_{4}\right)$. The mixtures were stirred for $30 \mathrm{~min}$ and sonicated for $10 \mathrm{~min}$ to ensure homogeneity in the water-glycerol system for freezing. Prior to measurement, $0.2 \mathrm{~mL}$ samples were loaded into an EPR tube and flash-frozen in liquid nitrogen.

Drying/Rehydration Treatment. The drying process for $\mathrm{Cu}$ WRAM was performed by placing the sample in a desiccator under ambient pressure for a period of 7 days, using silica gel as the desiccant. The rehydration was performed by placing the dried sample in a desiccator (without desiccant) with a vial of water to generate a humid environment. The sample was left for 7 days prior to measurement. For the Dowex resin, desiccation was observed to have little change on the resultant EPR spectrum. Instead, the sample was subjected to gentle evacuation on a Schlenk line, at room temperature, for a period of $2 \mathrm{~h}$. The rehydration process was the same as described for the CuWRAM sample. The degree of hydration was checked using EPR data (this work) and comparison with the "as received" samples.

Selective Unloading Study. Stock solutions of the $\mathrm{H}_{2} \mathrm{SO}_{4}$ and $\mathrm{NH}_{4} \mathrm{OH}$ eluents were prepared at varying concentrations, as required for the study $(0.05,0.1,0.5,1,1.5,2$, and $2.5 \mathrm{M})$. Samples of the respective resins $(0.05 \mathrm{~g})$ were weighed out and washed in a Büchner funnel with the chosen concentration of $\mathrm{H}_{2} \mathrm{SO}_{4}$ or $\mathrm{NH}_{4} \mathrm{OH}(20 \mathrm{~mL})$. Finally, the samples were washed with water and collected ready for EPR measurement.

CW EPR Spectroscopy. The X-band (9 GHz) CW-EPR spectra were recorded at $140 \mathrm{~K}$ on a Bruker EMX spectrometer operating at $100 \mathrm{kHz}$ field modulation, $10 \mathrm{~mW}$ microwave power using an ER 4119 HS cavity. Spectral simulations were performed using the EasySpin toolbox ${ }^{18}$ in Matlab, developed at ETH Zurich. 


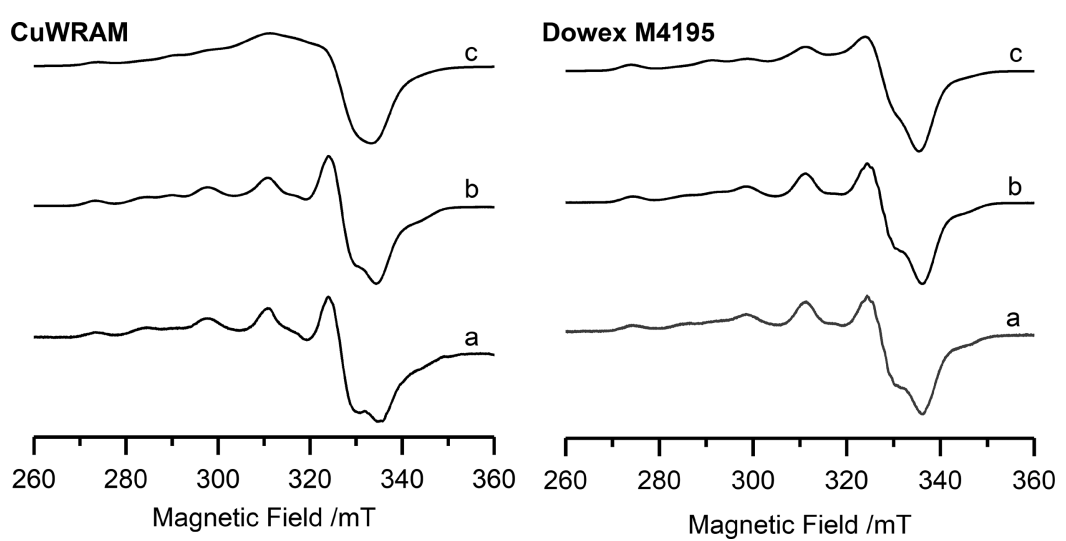

Figure 1. X-band CW EPR spectra (120 K) of CuWRAM and Dowex M4195 powders with increased loadings of copper: (a) 0.1, (b) 1 , and (c) 10 $\mathrm{mg} / \mathrm{g}$ of the polystyrene or silica resin.

UV Titration Experiments. Stock solutions of $\mathrm{H}_{2} \mathrm{SO}_{4}, \mathrm{CuSO}_{4}$, picolylamine, and bis-picolylamine were made up in deionized water. An initial solution $(20 \mathrm{~mL})$ was measured in a $50 \mathrm{~mL}$ tube, and varying aliquots of titrant solution were added. After mixing for $5 \mathrm{~min}$, an aliquot was taken to record the UV-vis spectra on a Cary 5000 UV-vis-NIR spectrometer, between 300 and 1200 at $5 \mathrm{~nm}$ intervals, and the $\mathrm{pH}$ was recorded of the solution. The spectra were processed using ReactLab Equilibria (JPlus Consulting Limited).

Equilibrium Uptake Experiments. Batch testing was carried out to determine the equilibrium adsorption capacity of the resins under varying $\mathrm{pH}$. CuWRAM or Dowex resin was measured into a centrifuge tube to which $35 \mathrm{~mL}$ of $\mathrm{CuSO}_{4}$ solution added and to this deionized water and $\mathrm{H}_{2} \mathrm{SO}_{4}$ were added (total $5 \mathrm{~mL}$ ). After $18 \mathrm{~h}$, a sample of solution was removed for analysis by ICP-OES, and the $\mathrm{pH}$ of the remaining solution was measured. The metal concentration of the blank (untreated) sample was compared against the concentration of the treated sample to calculate the amount of metal adsorbed.

Computations. Geometries were optimized using GAMESS-US (version 5, December, 2014, R1) ${ }^{19}$ at the DFT level of theory, in conjunction with the B3LYP functional ${ }^{20-23}$ (including the D3 empirical dispersion correction ${ }^{24}$ ), the Def2-SVP basis set, ${ }^{25}$ and the aqueous $\mathrm{SMD}^{26}$ implicit solvation model. Numerical frequencies were performed at the converged geometries to confirm stable energy minima on the potential energy surface as well as to obtain thermodynamic corrections to the electronic energies. More accurate energies were obtained by performing single-point energy calculations on the geometries obtained above, using the double-hybrid DSDPBEP86 functional, ${ }^{27,28}$ the triple- $\zeta$ Def2-TZVP basis set, ${ }^{25}$ and the SMD solvation model. ${ }^{26}$ These calculations were performed using ORCA (version 3.0.3), ${ }^{29}$ implementing the RIJCOSX approximation $^{30}$ to speed up the calculations. The double-hybrid calculations required an additional auxiliary correlation basis set of triple- $\zeta$ quality ${ }^{31}$ additionally, the RIJCOSX approximation required a second Coulomb-fitting auxiliary basis set, also of triple- $\zeta$ quality. ${ }^{32}$ Thermodynamic corrections obtained from the lower level of theory were added to the single-point energies obtained from the doublehybrid calculations to yield approximate aqueous Gibbs free energies $\left(G_{\mathrm{H}_{2} \mathrm{O}}\right)$. Initial conformations of the free picolylamine $(\mathrm{PA})$ and bispicolylamine (BPA) ligands were obtained using Open Babel's ${ }^{33}$ genetic algorithm-based conformer searching functionality. For each ligand, a maximum of 50 random conformers were generated, and their energies scored with the MMFF94 force field, ${ }^{34-38}$ as implemented in Open Babel. For each ligand, the lowest energy conformation was used as the starting point for DFT geometry optimizations. Finally, the DFT calculations were performed on the free complexes, rather than modeling the Dowex or WRAM supported complexes.

DFT Calculation of EPR Parameters. The resulting geometries were used to estimate EPR parameters in ORCA, ${ }^{29}$ using the hybrid
$\mathrm{PBE0}^{39}$ functional and basis set of EPRII ${ }^{40}$ for light atoms and the Core Properties all electron basis set for $\mathrm{Cu},{ }^{41}$ with spin-orbit effects accounted for in a mean field approach. ${ }^{42}$

\section{RESULTS AND DISCUSSION}

X-band EPR of $\mathrm{Cu}(\mathrm{II})$ Loaded Resins. The low-temperature X-band CW EPR spectra of the $\mathrm{Cu}(\mathrm{II})$ loaded CuWRAM and Dowex resins are shown in Figure 1. In each case, the samples were loaded with increasing amounts of copper $(0.1,1$, and $10 \mathrm{mg} / \mathrm{g}$ of the resin support using a $\mathrm{CuSO}_{4}$ solution). For clarity and comparison purposes, the EPR spectra shown in Figure 1 are normalized to the same intensity in order to highlight the complexity and speciation of $\mathrm{Cu}(\mathrm{II})$ centers present. The spectra are noticeably broadened, with reduced resolution at higher copper loadings, as expected due to spin-spin broadening. Despite the presence of the chelating $\mathrm{N}$-donor ligands (namely BPA and $\mathrm{PA}$ ), the ${ }^{14} \mathrm{~N}$ superhyperfine splittings are all but obscured from the powder EPR spectra, making the analysis and assignment of the contributing $\mathrm{Cu}(\mathrm{II})$ complexes more difficult.

Nevertheless, in all cases, a clear speciation of $\mathrm{Cu}$ (II) environments is evident in the spectra. This indicates that the $\mathrm{Cu}(\mathrm{II})$ ions exist in different coordination environments when anchored onto the resin support, contrary to the assumed uptake mechanism reported in the relevant literature. ${ }^{12,14}$ This agrees well with the partial protonation equilibria expected at low $\mathrm{pH}$, owing to competition between $\mathrm{H}_{2} \mathrm{SO}_{4} / \mathrm{CuSO}_{4}$ as a function of acid dissociation equilibria and operational $\mathrm{pH}$. Subtle changes in the coordination environment as a function of $\mathrm{pH}$ have been indicated based on DFT studies, ${ }^{43}$ and less favorable intermolecular ligand site coordinations, relative to the kinetic effect of chelation, are likely to become more important at the lower limits of the operational pH's. CuWRAM also possesses alternative potential donor sites in the support matrices, in the form of primary and secondary amines. However, these would be expected to be insignificant compared to the stronger bi/tridentate coordination from the abundant PA N-donor chelator groups. It should also be noted that partial protonation of the donor sites under the low $\mathrm{pH}$ conditions (as used in the extraction experiments) coupled with additional outer-sphere coordination may cause considerable change to the spin Hamiltonian parameters and the perceived $\mathrm{Cu}(\mathrm{II})$ speciation.

Clearly, deconvolution of the EPR spectra (Figure 1) is necessary in order to confidently identify the individual $\mathrm{Cu}(\mathrm{II})$-ligand environments present on the loaded resins. 
Therefore, in order to determine the distinct coordination centers present on these resins, a series of $\left[\mathrm{Cu}^{\mathrm{II}}(\mathrm{PA})_{x}\right]$ and $\left[\mathrm{Cu}^{\mathrm{II}}(\mathrm{BPA})_{x}\right]$ model complexes were prepared in aqueous solution by varying the ratio of $\mathrm{Cu}$ (II) ions to PA or BPA ligands. Analysis of the individual EPR spectra for the individual $\left[\mathrm{Cu}^{\mathrm{II}}(\mathrm{PA})_{x}\right]$ and $\left[\mathrm{Cu}^{\mathrm{II}}(\mathrm{BPA})_{x}\right]$ complexes formed should then enable one to deconvolute the spectra of the loaded samples (Figure 1).

$X-B$ and EPR Analysis of $\left[C u^{\prime \prime}(P A)_{x}\right]$ and $\left[C u^{\prime \prime}(B P A)_{x}\right]$ Model Complexes. The experimental X-band CW EPR spectra of aqueous solutions of $\mathrm{CuSO}_{4}$ titrated with increasing concentrations of the tridentate $\operatorname{di}(2$-picolylamine) chelator (BPA) are shown in Figure 2. Throughout the experiments,

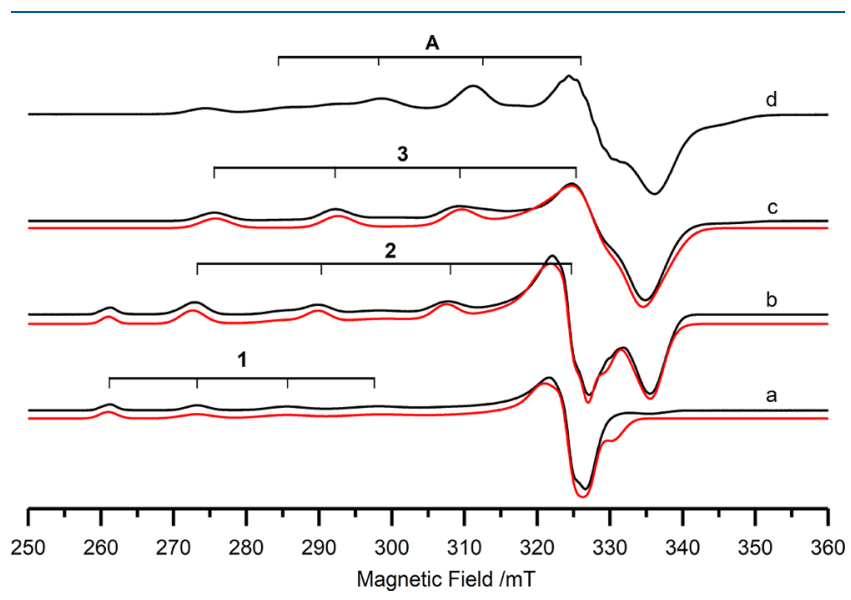

Figure 2. X-band CW EPR spectra $(120 \mathrm{~K})$ of Cu:BPA ratios of: (a) 1:0.1, (b) 1:1, and (c) 1:2, recorded in a water:glycerol mixture (3:2). For comparison, the CW EPR spectra of the Cu-loaded Dowex samples bearing $1 \mathrm{mg} / \mathrm{g}$ copper loading (Figure 1a,b) is shown in (d). Experimental $=$ black trace, simulated $=$ red trace.

glycerol was added as the glassing agent to the aqueous solution in order to produce a good polycrystalline EPR spectrum. Only the Cu:BPA ratios of $1: 0.1,1: 1$, and $1: 2$ are shown in Figure 2a-c. A more complete series of spectra showing the $\mathrm{Cu}$ :BPA ratios of 1:0.1, 1:05, 1:1, 1:2, 1:5, 1:10, and 1:50 are shown in the Supporting Information, from which it is clear that no changes occur in the EPR spectra for $\mathrm{Cu}: \mathrm{BPA}$ ratios higher than $1: 2$.

In all cases, well-defined EPR spectra characterized by axial symmetry can be observed (Figure $2 \mathrm{a}-\mathrm{c}$ ). At substoichiometric ratios of $\mathrm{Cu}: \mathrm{BPA}(1: 0.1)$, the spectrum is largely dominated by a single $\mathrm{Cu}$ (II) species, consistent with the presence of the expected and well reported pseudo-octahedral $\left[\mathrm{Cu}^{\mathrm{II}}\left(\mathrm{H}_{2} \mathrm{O}\right)_{6}\right]$ complex (Figure $\left.2 \mathrm{a}\right){ }^{44}$ A field-dependent strain effect is evident in the parallel hyperfine pattern, manifested as a progressive broadening of the peaks from the low field $m_{\mathrm{I}}=$ $-3 / 2$ to high field $m_{\mathrm{I}}=+3 / 2$ transitions. This effect is readily simulated using a correlated $g$ - and $A$-strain, arising from the distribution of $g / A$ values associated with weakly perturbed microenvironments in the sample. ${ }^{45}$ Hereafter this fully hydrated $\mathrm{Cu}$ (II) center will be referred to as species 1 .

At higher $\mathrm{Cu}$ :BPA ratios (1:1), a change occurs in the EPR spectrum (Figure $2 \mathrm{~b}$ ) due to the emergence of a second $\mathrm{Cu}$ (II) signal. This new signal can be easily assigned to a heteroleptic $\mathrm{Cu}$ (II) complex bearing a single coordinating BPA unit (i.e., $\left.\left[\mathrm{Cu}^{\mathrm{II}}(\mathrm{BPA})\right]\left(\mathrm{H}_{2} \mathrm{O}\right)_{m}\right)$, resulting from the ligation of one tridentate $\mathrm{BPA}$ ligand to the $\mathrm{Cu}(\mathrm{II})$ center and thereby reducing the $g_{\|} / A_{\|}$values relative to $\left[\mathrm{Cu}^{\mathrm{II}}\left(\mathrm{H}_{2} \mathrm{O}\right)_{6}\right]$. Hereafter this second $\left[\mathrm{Cu}^{\mathrm{II}}(\mathrm{BPA})\right]\left(\mathrm{H}_{2} \mathrm{O}\right)_{m}$ center will be referred to as species 2 (see Scheme 2). At the stoichiometric $\mathrm{Cu}$ :BPA ratio

Scheme 2. Structures of the Proposed $\left[\mathrm{Cu}^{\mathrm{II}}(\mathrm{PA})_{x}\right]$ and $\left[\mathrm{Cu}^{\mathrm{II}}(\mathrm{BPA})_{x}\right]$ Model Complexes<smiles>OC(O)C(O)(O)O</smiles>

$\left[\mathrm{Cu}\left(\mathrm{H}_{2} \mathrm{O}\right)_{6}\right]^{2+}$

1<smiles></smiles>

$\left[\mathrm{Cu}(\mathrm{PA})\left(\mathrm{H}_{2} \mathrm{O}\right)_{2}\right]^{2+}$

4<smiles></smiles>

$\left[\mathrm{Cu}(\mathrm{BPA})\left(\mathrm{H}_{2} \mathrm{O}\right)_{2}\right]^{2+}$ 2<smiles></smiles>

$\left[\mathrm{Cu}(\mathrm{PA})_{2}\right]^{2+}$ 5

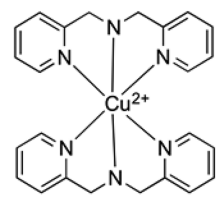

$\left[\mathrm{Cu}(\mathrm{BPA})_{2}\right]^{2+}$

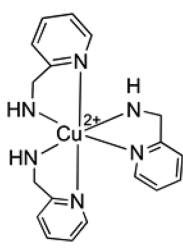

$\left[\mathrm{Cu}\left(\mathrm{PA}_{3}\right]^{2+}\right.$ of 1:1, an equilibrium exists since both species $\mathbf{1}$ and $\mathbf{2}$ are present in the frozen solution, as evident in the EPR spectrum (Figure $2 \mathrm{~b}$ ). Finally, for any $\mathrm{Cu}$ :BPA ratios higher than $1: 2$, a third signal becomes apparent in the spectrum (Figure 2c). This EPR signal has $g /{ }^{\mathrm{Cu}} A$ values which are distinctly different from those of species $\mathbf{1}$ and $\mathbf{2}$ and therefore can be assigned to the homoleptic $\mathrm{Cu}(\mathrm{II})$ complex (species 3) bearing two coordinating BPA ligands (i.e., $\left.\left[\mathrm{Cu}^{\mathrm{II}}(\mathrm{BPA})_{2}\right]\right)$. The observed behavior of this $\mathrm{Cu}(\mathrm{II})$ speciation in solution also agrees with the UV-vis titration data vide supra. The experimental spin Hamiltonian parameters for each species 1-3, as determined by simulations of the EPR spectra and by comparison with DFT derived values for the $\mathrm{Cu}(\mathrm{II})$ complexes, are given in Table 1.

To aid discussion of the reported $g_{\|} / A_{\|}$values, a PeisachBlumberg plot has been provided for reference to their correlation (ESI, Figure S6). The spin Hamiltonian parameters of $\left[\mathrm{Cu}^{\mathrm{II}}\left(\mathrm{H}_{2} \mathrm{O}\right)_{6}\right]$ (species 1) listed in Table 1 are entirely consistent with those widely reported in the literature. ${ }^{44}$ For species 2, the observed $g$ and ${ }^{\mathrm{Cu}} A$ values are also consistent with a $\mathrm{Cu}(\mathrm{II})$ ion bearing a tridentate N3-type ligand. ${ }^{46}$ Substitution of the $\mathrm{H}_{2} \mathrm{O}$ ligands for strongly coordinating, electron-rich nitrogen atoms, is responsible for the expected decrease in the $g_{\|} / A_{\|}$values, and these values are typical for square-based pyramidal $\mathrm{Cu}(\mathrm{II})$ geometry. ${ }^{46}$ The rather large $A_{\|}$values for species 2 may indicate the inner-sphere coordination of a sulfate counterion, as previously suggested, which would have a small effect in $g_{\|}$, but a marked increase in $A_{\|}{ }^{44}$ The $g_{\|} / A_{\|}$values of the coordinatively saturated $\left[\mathrm{Cu}^{\mathrm{II}}(\mathrm{BPA})_{2}\right]$ complex (species 3 ) are only slightly lower than those for species 2 (Table 2). However, this species has been reported to exist in both pseudo-octahedral and squarebased pyramidal geometries, where the latter is associated with a noncoordinating pyridyl nitrogen (see Scheme A, Supporting Information, for proposed structure).$^{47}$ Nevertheless, the reported ${ }^{\mathrm{Cu}} A_{\|}$values suggest the environment is closer to pseudo-octahedral geometry with an N4-equatorial donor set. $^{46}$ 
Table 1. Experimental and Calculated $g$ and ${ }^{\mathrm{Cu}} A$ Spin Hamiltonian Parameters for $\left[\mathrm{Cu}{ }^{\mathrm{II}}(\mathrm{BPA})_{x}\right]$ and $\left[\mathrm{Cu}^{\mathrm{II}}(\mathrm{PA})_{x}\right] \mathrm{Complexes}^{a}$

\begin{tabular}{|c|c|c|c|c|c|c|c|c|}
\hline species & & $g_{x}^{b}$ & $g_{y}^{b}$ & $g_{z}{ }^{c}$ & $\left|A_{x}\right|^{d}$ & $\left|A_{y}\right|^{d}$ & $\left|A_{z}\right|^{e}$ & UV abs \\
\hline \multirow[t]{2}{*}{1} & $\exp$ & 2.094 & 2.072 & 2.418 & NR & NR & 413 & 710 \\
\hline & DFT & 2.099 & 2.126 & 2.303 & 121 & 192 & 484 & \\
\hline \multirow[t]{2}{*}{2} & $\exp$ & 2.062 & 2.065 & 2.262 & 46 & 70 & 556 & 610 \\
\hline & DFT & 2.049 & 2.075 & 2.204 & 36 & 55 & 557 & \\
\hline \multirow[t]{2}{*}{3} & $\exp$ & 2.046 & 2.072 & 2.243 & 30 & 42 & 535 & $710(890)$ \\
\hline & DFT & 2.060 & 2.062 & 2.204 & 30 & 43 & 542 & \\
\hline \multirow[t]{2}{*}{4} & $\exp$ & 2.061 & 2.071 & 2.303 & 23 & 45 & 530 & 690 \\
\hline & DFT & 2.046 & 2.054 & 2.170 & 44 & 70 & 629 & \\
\hline \multirow[t]{2}{*}{5} & $\exp$ & 2.050 & 2.058 & 2.229 & 34 & 62 & 593 & 590 \\
\hline & DFT & 2.040 & 2.052 & 2.153 & 18 & 66 & 603 & \\
\hline 6 & $\exp$ & 2.064 & 2.064 & 2.254 & 45 & 45 & 515 & 590 \\
\hline A & $\exp$ & 2.038 & 2.038 & 2.213 & 55 & 55 & 436 & - \\
\hline B & $\exp$ & 2.050 & 2.050 & 2.220 & 45 & 45 & 410 & - \\
\hline
\end{tabular}

${ }^{a} A_{x}$ and $A_{y}$ hyperfine components were not resolved and therefore are estimated. Experimental UV absorption data reported in nm. NR $=$ not resolved. ${ }_{g} g$ value: \pm 0.003 . ${ }^{c} g$ value: $\pm 0.003 .{ }^{d} A_{i}$ magnitude value $\pm 10 .{ }^{e} A_{i}$ magnitude value \pm 3 .

Table 2. Log $K$ Values Obtained by UV-vis for the $\mathrm{Cu}(\mathrm{II})$ BPA and -PA Speciation Study

\begin{tabular}{lll}
\multicolumn{1}{c}{ step } & $\log K$ & error \\
$\mathrm{Cu}+\mathrm{PA} \rightarrow \mathrm{CuPA}$ & 7.853 & 0.067 \\
$\mathrm{CuPA}+\mathrm{PA} \rightarrow \mathrm{CuPA}_{2}$ & 5.725 & 0.062 \\
$\mathrm{CuPA}+\mathrm{PA} \rightarrow \mathrm{CuPA}_{3}$ & 1.510 & 0.012 \\
$\mathrm{Cu}+\mathrm{BPA} \rightarrow \mathrm{CuBPA}$ & 6.942 & 0.132 \\
$\mathrm{CuBPA}+\mathrm{BPA} \rightarrow \mathrm{CuBPA}_{2}$ & 4.156 & 0.070 \\
\hline
\end{tabular}

The above series of ligand titration experiments performed for the $\left[\mathrm{Cu}^{\mathrm{II}}(\mathrm{BPA})_{x}\right]$ complexes were then repeated using the 2-picolylamine (PA) ligands. The resulting X-band CW EPR spectra for the resulting $\left[\mathrm{Cu}^{\mathrm{II}}(\mathrm{PA})_{x}\right]\left(\mathrm{H}_{2} \mathrm{O}\right)_{m}$ complexes formed by increasing the $\mathrm{Cu}: \mathrm{PA}$ ratios are shown in Figure $3 \mathrm{a}-\mathrm{c}$. The more complete series of spectra showing the Cu:PA ratios of 1:0.1, 1:05, 1:1, 1:2, 1:5, 1:10, and 1:50 are given in the Supporting Information.
Once again, all the EPR spectra reveal an axial environment at this operating frequency (X-band). At the lowest $\mathrm{Cu}: \mathrm{PA}$ ratio studied here (1:0.1), the presence of the fully hydrated $\mathrm{Cu}$ (II) center (species $\mathbf{1}$ ) is clearly visible (shown in the Supporting Information). At the stoichiometric $\mathrm{Cu}$ :PA ratio of $1: 1$, two $\mathrm{Cu}(\mathrm{II})$ centers are visible in the spectrum (Figure 3a) arising from a mix of species 1 and an additional new center hereafter labeled species $\mathbf{4}$. We assign this latter species 4 to the heteroleptic complex $\left[\mathrm{Cu}^{\mathrm{II}}(\mathrm{PA})\right]\left(\mathrm{H}_{2} \mathrm{O}\right)_{m}$ arising from coordination of one PA ligand to $\mathrm{Cu}(\mathrm{II})$. At slightly higher ratios (1:2), another $\mathrm{Cu}(\mathrm{II})$ center can be readily identified in the spectrum (Figure $3 \mathrm{~b}$ ), labeled species 5 . This species can be easily assigned to the bis-adduct complex $\left[\mathrm{Cu}^{\mathrm{II}}(\mathrm{PA})_{2}\right]$ $\left(\mathrm{H}_{2} \mathrm{O}\right)_{m}$ formed by two PA ligands binding to the $\mathrm{Cu}(\mathrm{II})$ ion. A partly resolved ${ }^{14} \mathrm{~N}$ superhyperfine pattern is also evident in the perpendicular region of the spectrum, indicating nitrogen coordination, but lacking sufficient resolution to extract the number of coordinated ${ }^{14} \mathrm{~N}$ nuclei (Figure $3 \mathrm{~b}$ ). At the highest

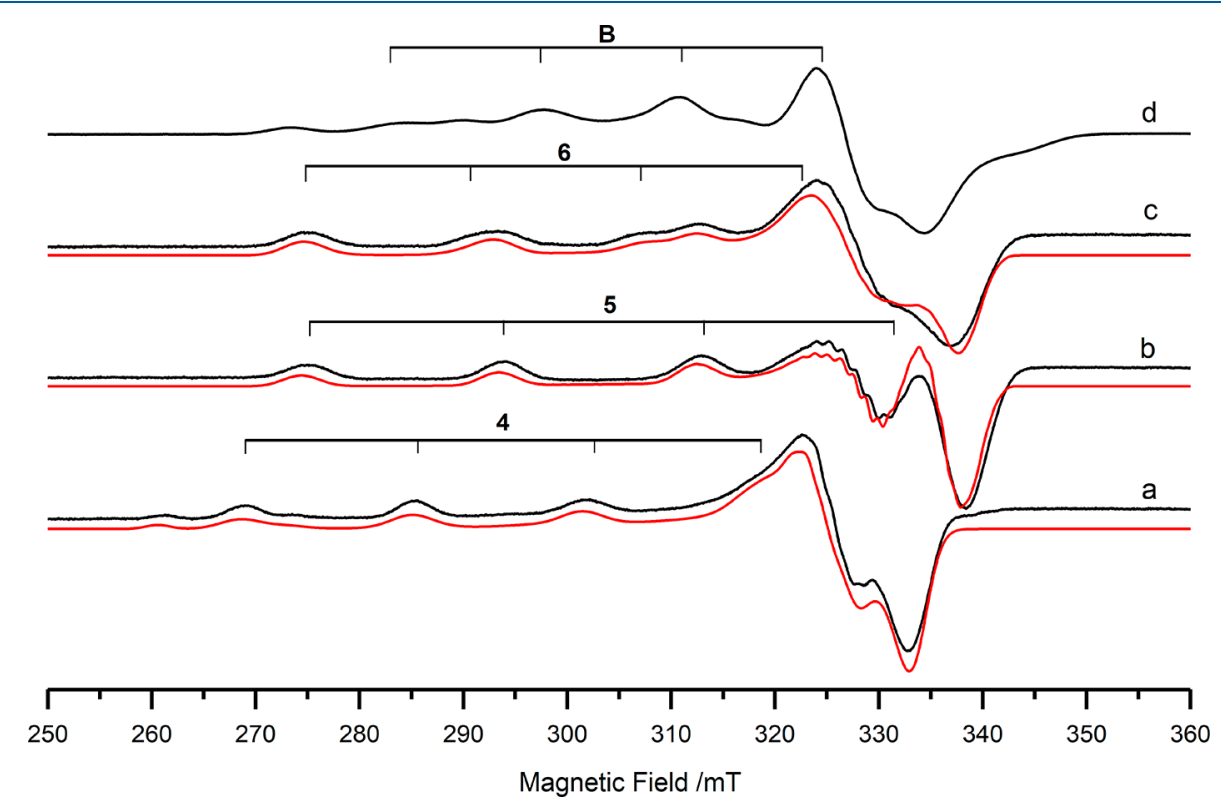

Figure 3. X-band CW EPR spectra (120 K) of Cu:PA ratios of: (a) 1:1, (b) 1:2, and (c) 1:50, recorded in a water:glycerol mixture (3:2). For comparison, the CW EPR spectra of the Cu-loaded CuWRAM samples bearing $1 \mathrm{mg} / \mathrm{g}$ copper loading (Figure $1 \mathrm{~b})$ is shown in $(\mathrm{d})$. Experimental = black trace, simulated $=$ red trace. 
$\mathrm{Cu}: \mathrm{PA}$ ratios studied here (1:50), an additional signal is just apparent in the EPR spectrum (low intensity), most notably on the low field side of the $m_{\mathrm{I}}=+3 / 2$ transition (Figure 3c). We assign this to the formation of the triadduct complex $\left[\mathrm{Cu}^{\mathrm{II}}(\mathrm{PA})_{3}\right]$ (species 6), comparable to the isostructural $\left[\mathrm{Ni}(\mathrm{PA})_{3}\right]^{2+}$ complex and observed for other first-row transition metals. ${ }^{48}$ However, it should be noted that the bisadduct $\left[\mathrm{Cu}^{\mathrm{II}}(\mathrm{PA})_{2}\right]\left(\mathrm{H}_{2} \mathrm{O}\right)_{m}$ complex remains the more abundant species even at these higher ratios (Figure $3 \mathrm{c}$ ). Therefore, extraction of the exact spin Hamiltonian parameters for species 6 is difficult owing to the overlapping features from species $\mathbf{4}$ and $\mathbf{5}$, so only approximate values are given in Table 1. Furthermore, in support of these EPR findings, complementary UV-vis measurements also indicate the presence of a third metal-ligand species at high ligand ratios.

The spin Hamiltonian parameters obtained by simulation of the experimental spectra for species 4,5 , and 6 (assigned to $\left[\mathrm{Cu}^{\mathrm{II}}(\mathrm{PA})\right]\left(\mathrm{H}_{2} \mathrm{O}\right)_{m}, \quad\left[\mathrm{Cu}^{\mathrm{II}}(\mathrm{PA})_{2}\right]\left(\mathrm{H}_{2} \mathrm{O}\right)_{m}$, and $\left.\left[\mathrm{Cu}^{\mathrm{II}}(\mathrm{PA})_{3}\right]\right)$ are listed in Table 1. Similar to the BPA adducts, the PA adducts can be easily distinguished based on the decreasing $g_{\|}$ and ${ }^{\mathrm{Cu}} A_{\|}$values (relative to the hydrated $\left[\mathrm{Cu}^{\mathrm{II}}\left(\mathrm{H}_{2} \mathrm{O}\right)_{6}\right]$ complex), indicating an increasing degree of covelancy due to the strong interactions of the $\mathrm{Cu}$ (II) ion with the neutral, nitrogen-donor sites on the PA ligands. In particular, the $g_{\|}$and ${ }^{\mathrm{Cu}} A_{\|}$values for species $\mathbf{4}$ and $\mathbf{5}$ are indicative of a square-planar geometry, with PA ligands coordinating in the equatorial plane. ${ }^{46}$ The slightly higher value of $g_{\|}$for species 4 is likely due the asymmetric bonding interactions in the equatorial plane, due to the incomplete coordination sphere. The assignment of the largely square-planar geometry, despite the high value for $g_{\|}$, is also supported by the progressively blueshifted $\mathrm{d}-\mathrm{d}$ band transitions observed with increasing ligand ratios in UV-vis titrations reported vide infra. Finally, the spin Hamiltonian parameters for species $\mathbf{6}$ are consistent with a pseudo-octahedral geometry, generally unfavored by $\mathrm{Cu}$ (II) due to its tendency to weakly coordinate in the axial positions. However, the decrease in magnitude of ${ }^{\mathrm{Cu}} A_{\|}$and the increase in $g_{\|}$, compared to species $\mathbf{5}$, are consistent with the lower spin delocalization in the equatorial plane due to interactions in the axial positions. The proposed structures for species 4-6 are shown in Scheme 2.

UV-vis of Metal Titration with Ligand. A similar experiment to that described above for the model $\mathrm{Cu}$ (II) complexes using EPR was repeated using UV-vis spectroscopy. Initially, the $\mathrm{CuSO}_{4}$ solution $(0.01 \mathrm{M})$ was titrated with an increasing volume of a PA solution $(0.1 \mathrm{M})$. As the PA is added, at 1 equiv, a rapid change occurs in the spectrum with the appearance of a band at $710 \mathrm{~nm}$. From 2 equiv of ligand, the band shifts to $690 \mathrm{~nm}$. The ligand itself shows no absorption above $500 \mathrm{~nm}$ (Figure 4.)

Closer analysis of the data in Figure 4 shows that the titration can be broken down into at least two distinct processes (with associated isosbestic points) that occur from 0 to 1 equiv and 1 to 2 equiv of added ligand (see Supporting Information). The UV-vis data were then modeled to understand the underlying processes. A two-step model (i.e., $\mathrm{Cu}+\mathrm{L} \rightarrow \mathrm{CuL} ; \mathrm{CuL}+\mathrm{L} \rightarrow \mathrm{CuL}_{2}$ ) was first used to process the titration data, but the fit was poor (see Supporting Information). Hence a three-step model, which included a third step (including formation of $\mathrm{CuL}_{3}$ ), was used, and a much better fit was obtained. The absorption spectra for the third species (i.e., $\mathrm{CuPA}_{3}$ ) was predicted to be very similar to the $\mathrm{CuL}_{2}$ species (i.e., $\mathrm{CuPA}_{2}$ ) in the $590 \mathrm{~nm}$ region with an

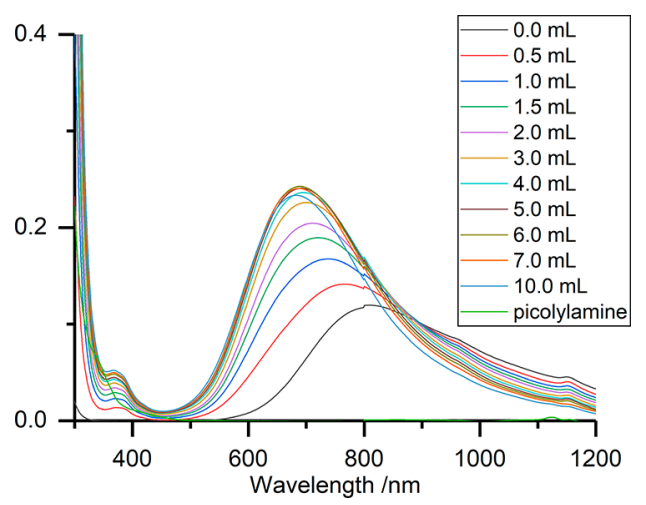

Figure 4. UV-vis spectra of a $\mathrm{CuSO}_{4}$ solution bearing an increasing concentration of PA ligand.

additional shoulder at $890 \mathrm{~nm}$. The $\log K$ values (Table 2) reveal that the formation of $\mathrm{CuPA}$ and $\mathrm{CuPA}_{2}$ is more favorable compared to formation of $\mathrm{CuPA}_{3}$, in agreement with the EPR data where the species were identified at low ratios. The titration was also carried out in reverse, whereby a $\mathrm{Cu}$ (II) solution was added to the PA solution, with a similar trend and observations obtained.

The stability of the $\mathrm{Cu}$ (II) complexes in acidic environments was also tested. When a solution bearing a $\mathrm{Cu}: \mathrm{PA}$ ratio of 1:2.25 was titrated with $\mathrm{H}_{2} \mathrm{SO}_{4}$, a clear change in chelation from $\mathrm{CuPA}_{2}$ to unchelated free $\mathrm{Cu}$ was evident in the UV-vis spectra (see Supporting Information). As the $\mathrm{pH}$ decreased below 4, the $\mathrm{CuPA}_{2}$ species rapidly diminished in intensity until at $\mathrm{pH}=2$ no signal from this complex was detected. Similarly, the CuPA species also diminished from $\mathrm{pH}=2.5$ until at ca. $\mathrm{pH}=1$ there is virtually no detectable chelated $\mathrm{Cu}$ (II) with the UV-vis spectra matching that of the $\mathrm{CuSO}_{4}$ solution (Supporting Information). When a similar titration was carried out with the $\mathrm{Cu}: \mathrm{BPA}$ complexes, the $\mathrm{CuBPA}_{2}$ species was found to gradually decrease in concentration with increasing $\mathrm{pH}$. However, in this case, the CuBPA complex remains stable even up to $\mathrm{pH}=0.8$ and can still be observed in the spectra at $\mathrm{pH}=0.5$. This indicates that the BPA ligand forms an extremely stable complex with the $\mathrm{Cu}$ (II) ions which then cannot be easily displaced in acidic media (i.e., via protonation of the amine nitrogen), whereas the $\mathrm{Cu}: \mathrm{PA}$ complex is disrupted around $\mathrm{pH}=2$, likely caused by the protonated amine ligand-copper complex being less favorable (i.e., the proton more favorably interacts with the ligand that then $\mathrm{Cu}(\mathrm{II}))$.

A similar UV-vis study was performed for the titration of $\mathrm{CuSO}_{4}$ with the BPA ligand. As with the PA addition, the titration was carried out by titrating a copper sulfate solution $(10 \mathrm{~mL}, 0.01 \mathrm{M})$ with a solution of BPA $(0.1 \mathrm{M})$. Initially, a two-step reaction model was used where the copper and ligand solution spectra were fixed, which resulted in a very poor fit. The model was adjusted with a fixed copper solution spectra and an unknown ligand spectra (ESI, Figure S12). This gave an improved but still relatively poor fit with a similar issue to that previously indicated; whereby, a negative peak was required in the ligand spectra to fit at high ligand excess (at low wavelengths). The corresponding UV-vis spectra have been omitted due to the poor modeling. Nevertheless, the $\log K$ values indicate a favorable formation of $\mathrm{CuBPA}$, and $\mathrm{CuBPA}_{2}$, in comparison to the values derived from the PA titrations. 
Assignment of $\mathrm{Cu}$ (II) Species on Loaded Resins. Once the model $\mathrm{Cu}$ (II) species were characterized by EPR and confirmed by UV-vis, analysis and deconvolution of the experimental CuWRAM and Dowex EPR spectra (Figure 1) become more straightforward. The first point to note is that there is no evidence for the hydrated $\left[\mathrm{Cu}^{\mathrm{II}}\left(\mathrm{H}_{2} \mathrm{O}\right)_{6}\right]$ complex (species 1) in these spectra (Figure 1). This is not unexpected, particularly owing to the relatively hydrophobic framework on which the chelating groups are anchored. In the Dowex case, species 2 and 3 can be identified by simulation of the experimental spectrum (Figure 2d), although the poor resolution of the spectrum prevents an accurate quantitative estimate of the relative abundance of each species. The observed modulation in the perpendicular region of the EPR spectrum for the Dowex sample (Figure $2 \mathrm{~d}$ ) arises from ${ }^{14} \mathrm{~N}$ superhyperfine interactions, further supporting the assignment to species 2 and 3 . However, despite the identification of species $\mathbf{2}$ and 3 in the EPR spectrum of the $\mathrm{Cu}$ loaded Dowex sample, an unidentified EPR signal remains. This is highlighted as species $\mathbf{A}$ in Figure $2 \mathrm{~d}$. An estimate of the spin Hamiltonian parameters for this unidentified $\mathrm{Cu}$ (II) center (hereafter labeled species A) is listed in Table 1 . The $g_{\|}$and $A_{\|}$values of 2.21 and $436 \mathrm{MHz}$, respectively, suggest a tetragonal perturbed square-planar complex, ${ }^{46}$ which would be consistent with a highly distorted, weakly bound environment that may arise due to the protonation equilibria of the ligand, or more likely an intermolecular coordination mode by neighboring BPA ligands (Scheme 1). In other words, two BPA ligands may interact with the $\mathrm{Cu}$ (II) ion, but involving different numbers of coordinating $\mathrm{N}$-nuclei. Hence we assign this to a more weakly coordinating intermolecular $\left[\mathrm{Cu}{ }^{\mathrm{II}}(\mathrm{BPA})_{x}(\mathrm{BPA})_{y}\left(\mathrm{H}_{2} \mathrm{O}\right)_{n}\right]$ complex, as opposed to the intramolecular $\left[\mathrm{Cu}^{\mathrm{II}}(\mathrm{BPA})\right]\left(\mathrm{H}_{2} \mathrm{O}\right)_{m}$ and $\left[\mathrm{Cu}^{\mathrm{II}}(\mathrm{BPA})_{2}\right]$ complexes (species 2 and 3 ). The presence of the coordinated water is evidenced from the dehydration studies vide infra.

For the CuWRAM sample, the experimental EPR spectrum (Figure 3d) could be simulated and deconvoluted knowing the spin Hamiltonian parameters for the model complexes species 4-6. It is evident from the simulations that the spectrum is dominated by signals assigned to the bis- and tris-adducts (species 5 and 6), with no evidence for any substantial contributions coming from $\left[\mathrm{Cu}^{\mathrm{II}}\left(\mathrm{H}_{2} \mathrm{O}\right)_{6}\right]$ or $\left[\mathrm{Cu}^{\mathrm{II}}(\mathrm{PA})\right]$ $\left(\mathrm{H}_{2} \mathrm{O}\right)_{m}$ complexes (species 1 and 4). Similar to the Dowex case, an additional unassigned signal, not characterized by the $g /{ }^{\mathrm{Cu}} A$ values of the model complexes (species 4-6), can be seen in the spectrum (labeled B in Figure 3d). An estimate of the spin Hamiltonian parameters for this species is given in Table 1 (labeled species B). Once again, the $g_{\|}$and $A_{\|}$values of 2.22 and $410 \mathrm{MHz}$ are similar to that observed on the Dowex sample, and this species can also be assigned either to coordination to the PA ligands with partially protonated chelating sites, dependent on $\mathrm{pH}$ and equilibrium acidities, or the different degrees of intermolecular $\mathrm{N}$-coordination by neighboring PA. In solution, one, two, or three bidentate PA ligands coordinate to the $\mathrm{Cu}(\mathrm{II})$, but on the CuWRAM sample, it appears that mono- and bidentate coordination is possible (not reproduced in the model solution phase experiments).

Dehydration/Rehydration Studies. According to the above findings, the loaded $\mathrm{Cu}(\mathrm{II})$ resins contain $\left[\mathrm{Cu}^{\mathrm{II}}(\mathrm{PA})_{x}\right]$ and $\left[\mathrm{Cu}^{\mathrm{II}}(\mathrm{BPA})_{x}\right]$ complexes, with intra- and intermolecular coordination of the ligands anchored to the resins and likely bearing coordinated water molecules within the complex. To explore the latter point further, the resin samples were subjected to a series of dehydration and rehydration experiments. For CuWRAM, the sample was dried over silica gel for several hours and subsequently rehydrated in a controlled atmosphere for several hours. The resulting EPR spectra are shown in Figure 5. Clear differences can be observed between

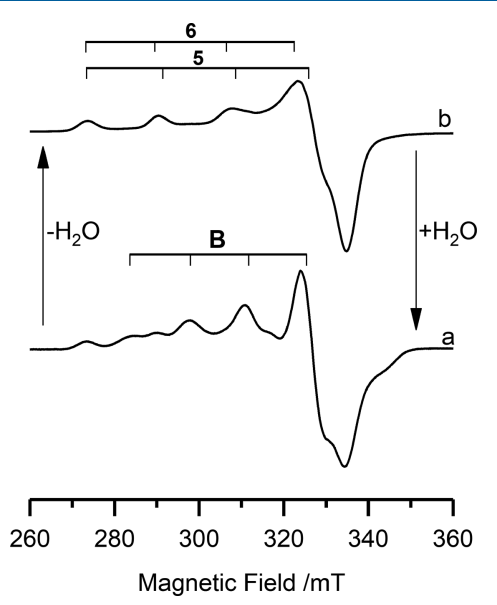

Figure 5. X-band CW EPR spectra $(120 \mathrm{~K})$ of the loaded CuWRAM sample $(1 \mathrm{mg} / \mathrm{g}$ loaded resin) undergoing repeated dehydrationrehydration treatments: (a) hydrated material and (b) dehydrated material.

the hydrated and dehydrated spectra, and the results were found to be completely reversible. This procedure was repeated several times, to ensure the observed reversibility was not associated with any changes in the polymer structure (e.g., framework degradation or shrink-swell changes) and instead is indeed associated with the loss of water. It is clear that only the signal assigned to species $\mathbf{B}$ on the CuWRAM sample is affected, which suggests that this intermolecular $\left[\mathrm{Cu}(\mathrm{PA})_{x}(\mathrm{PA})_{y}\left(\mathrm{H}_{2} \mathrm{O}\right)_{n}\right]$ complex bears strongly coordinated $\mathrm{H}_{2} \mathrm{O}$ molecules in the inner sphere.

The EPR spectrum of the resulting dried sample (Figure 5b) is broadened, but contains observable signals associated with species $5\left(\left[\mathrm{Cu}^{\mathrm{II}}(\mathrm{PA})_{2}\right]\right)$ and $6\left(\left[\mathrm{Cu}^{\mathrm{II}}(\mathrm{PA})_{3}\right]\right)$. Because the spin Hamiltonian parameters for these species did not change upon drying, it suggests any coordinated water is only weakly bound and does not affect the EPR signals. Following dehydration of species B $\left[\mathrm{Cu}^{\mathrm{II}}(\mathrm{PA})_{x}(\mathrm{PA})_{y}\left(\mathrm{H}_{2} \mathrm{O}\right)_{n}\right]$, the spin Hamiltonian parameters of the resulting $\left[\mathrm{Cu}^{\mathrm{II}}(\mathrm{PA})_{x}(\mathrm{PA})_{y}\right]$ must bear close resemblance to those of species $\mathbf{5}$ and $\mathbf{6}$, since no new features were observed in the spectrum and the overall integrated $\mathrm{Cu}(\mathrm{II})$ signal intensity did not change. It is unlikely that the dehydrated $\left[\mathrm{Cu}^{\mathrm{II}}(\mathrm{PA})_{x}(\mathrm{PA})_{y}\right]$ rearranges into species 5 or $\mathbf{6}$, since upon rehydration, the signal from species $\mathbf{B}$ reappears, indicating a reversible $\left[\mathrm{Cu}^{\mathrm{II}}(\mathrm{PA})_{x}(\mathrm{PA})_{y}\left(\mathrm{H}_{2} \mathrm{O}\right)_{n}\right] \leftrightarrow$ $\left[\mathrm{Cu}^{\mathrm{II}}(\mathrm{PA})_{x}(\mathrm{PA})_{y}\right]$ process, and the more coordinatively saturated species 5 and $\mathbf{6}$ will not be so influenced by water.

The above experiments were also repeated for the Dowex sample, wherein a similar species A signal was identified in the EPR spectrum. The resulting dehydration-rehydration spectra are shown in the Supporting Information. It should be noted that in this case, it proved more difficult to dehydrate the sample and gentle thermal-evacuation was required to elicit a change in the spectra. Although the changes in the spectra were less pronounced compared to the CuWRAM sample (Figure 4), nevertheless an analogous reversible behavior was 
once again identified, suggesting that species $\mathbf{A}$ in the Dowex sample can also be assigned to a $\mathrm{Cu}$ (II) complex bearing coordinated water molecules, that is, $\left[\mathrm{Cu}^{\mathrm{II}}(\mathrm{BPA})_{x}(\mathrm{BPA})_{y}\left(\mathrm{H}_{2} \mathrm{O}\right)_{n}\right] \leftrightarrow\left[\mathrm{Cu}^{\mathrm{II}}(\mathrm{BPA})_{x}(\mathrm{BPA})_{y}\right]$.

Selective $\mathrm{Cu}(\mathrm{II})$ Unloading Studies. Typically, the adsorbed $\mathrm{Cu}$ (II) ions that are collected by the resins during the elution cycle are subsequently released by washing with concentrated acid (1-2 $\left.\mathrm{M} \mathrm{H}_{2} \mathrm{SO}_{4}\right)$ which protonate the nitrogen sites. Acid washing of the Dowex resin is insufficient to remove all of the $\mathrm{Cu}$ (II) ions, and in such cases, the sample requires washing with a concentrated ammonia solution (1-2 $\mathrm{M} \mathrm{NH}_{4} \mathrm{OH}$ ) to fully release the metal. We therefore undertook a series of elution experiments using increasing concentrations of acid (or base) to explore any variation in binding strength, as monitored by changes in the EPR spectra. The resulting EPR spectra for the loaded CuWRAM sample before and after washing with increasing concentration of $\mathrm{H}_{2} \mathrm{SO}_{4}$ are shown in Figure 6.

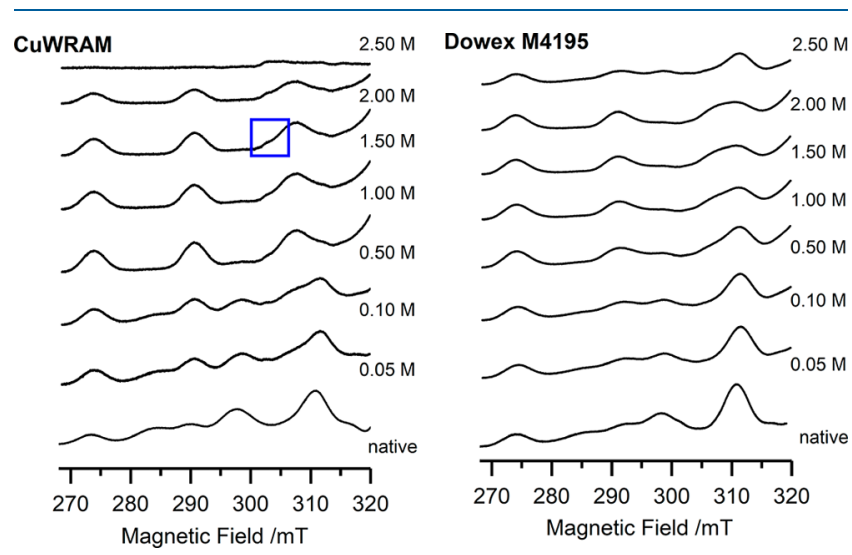

Figure 6. X-band CW EPR spectra $(120 \mathrm{~K})$ of the loaded CuWRAM and Dowex M4195 samples (1 mg/g loaded resins), after washing with increasing concentrations of $\mathrm{H}_{2} \mathrm{SO}_{4}: 0.05,0.1,0.5,1,1.5,2$, and $2.5 \mathrm{M}$.

The overall EPR signal intensity steadily decreases as the concentration of acid increases. At the highest concentrations studied here $(2.5 \mathrm{M})$, virtually all of the $\mathrm{Cu}$ (II) ions have been removed from the sample. However, one striking observation from the spectra is the rapid and immediate loss of species $\mathbf{B}$ from the CuWRAM sample at low $\mathrm{H}_{2} \mathrm{SO}_{4}$ concentrations. At $0.5 \mathrm{M} \mathrm{H}_{2} \mathrm{SO}_{4}$ concentration, virtually all of species $\mathbf{B}$ has been removed from the sample (Figure $5 \mathrm{~d}$ ). At slightly higher concentrations (from 0.5 to $1.5 \mathrm{M}$ ), the EPR spectra are reminiscent of the signals identified in the dehydrated sample (Figure $5 \mathrm{~b}$ ) and indicate that only the bis-adduct species 5 and tris-adduct species 6 remain. At $1.5 \mathrm{M} \mathrm{H}_{2} \mathrm{SO}_{4}$ concentration, a small shoulder emerges on one of the parallel hyperfine lines (labeled with a blue box in Figure 6, low field $\mathrm{m}_{I}=+1 / 2$ transition at $312 \mathrm{mT}$ ), and based on the simulation, it appears that this feature is associated with species 6. This result suggests that, following elution of the more labile species $\mathbf{B}$, the bis-adduct is then preferentially removed before the more highly coordinated triadduct species 6 (although the resolution of the spectra were insufficient to accurately quantify this observation). Nevertheless, this observation would be a reasonable expectation associated with the $\mathrm{Cu}(\mathrm{II})$ speciation present on these sample, and these results also fit with the assigned coordinations from solution measurements and UVvis data vide infra.

A similar series of experiments were performed by washing the Dowex sample with increasing concentrations of $\mathrm{H}_{2} \mathrm{SO}_{4}$. The resulting spectra are shown in the Supporting Information. The results are very similar to the above findings observed for the CuWRAM sample. In particular, species A was selectively and preferentially removed at the lower acid concentrations, while the appearance of a small shoulder at $312 \mathrm{mT}$ (assigned to the fully coordinated species 3 ), relative to the signal from species $\mathbf{2}$ at higher concentrations, suggests that the heteroleptic complex $\mathbf{2}$ is eluted before the homoleptic complex 3. It should be noted that even at the highest concentrations of acid used $(2.5 \mathrm{M})$, residual $\mathrm{Cu}(\mathrm{II})$ ions remain attached to the resin (Supporting Information), in agreement with reported kinetics measurements on Dowex relative to CuWRAM. ${ }^{3}$ This clearly indicates that protonation of the BPA chelate binding sites is insufficient to release species $\mathbf{2}$ and 3 from the resin. Therefore, the $\mathrm{Cu}$ loaded resin samples were washed with an aqueous ammonia solution of increasing concentrations $(0.05-2.5 \mathrm{M})$, and the resulting spectra are shown in the Supporting Information. An identical trend to that observed above with acid washing was found in the ammonia washed samples, with the residual signal visible at $2.5 \mathrm{M} \mathrm{NH}_{4} \mathrm{OH}$ (aq.) concentration having parameters typical of species 3 .

Equilibrium batch uptake measurements were carried out to determine the equilibrium $\mathrm{Cu}$ (II) capacity at varying $\mathrm{pH}$. The resulting capacity data are shown in Figure 7. It is clear from
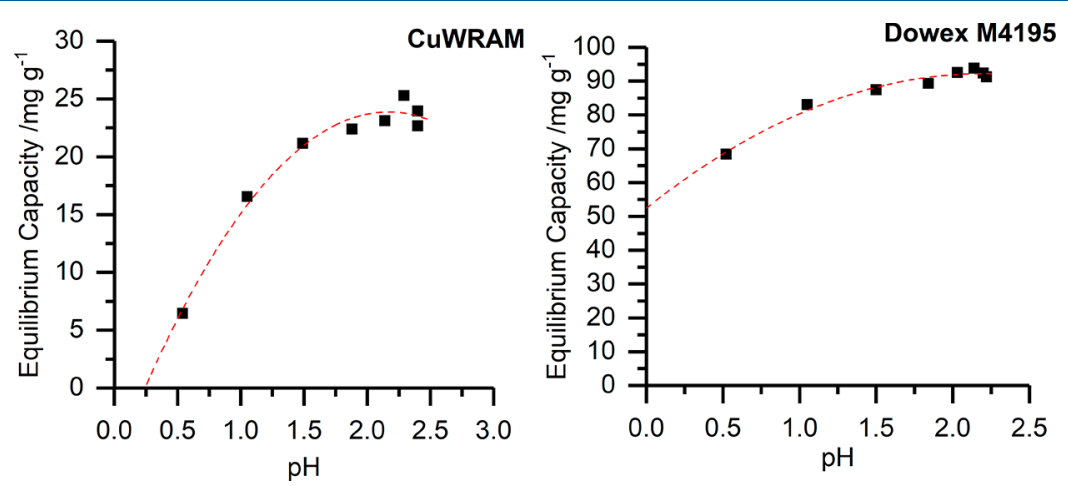

Figure 7. Copper capacity of CuWRAM measured using $0.4 \mathrm{w} / \mathrm{v} \%$ resin, and Dowex M4195 measured using $0.2 \mathrm{w} / \mathrm{v} \%$. Initial solution of $500 \mathrm{ppm}$ copper, $\mathrm{pH}$ adjusted using $\mathrm{H}_{2} \mathrm{SO}_{4}$. Error in equilibrium capacity measurements $\pm 2 \mathrm{mg} \mathrm{g}^{-1}$. Error in $\mathrm{pH}$ measurements $\pm 0.01 ; R^{2}(\mathrm{CuWRAM})=$ $0.980 ; R^{2}$ (Dowex M4195) $=0.978$. 
the data that the silica-based CuWRAM material has a much lower capacity than the polymeric Dowex material, as expected. The $\mathrm{Cu}(\mathrm{II})$ capacity profile for the two resins is also different. The CuWRAM sample shows a rapid reduction in capacity as the $\mathrm{pH}$ is reduced below 2 , with only $6 \mathrm{mg} / \mathrm{g}$ capacity at $\mathrm{pH}=$ 0.5 which is $28 \%$ of the capacity at $\mathrm{pH}=2$. Below $\mathrm{pH}=0.5$, the capacity rapidly decreases, similar to the EPR data which revealed a facile loss in the $\mathrm{Cu}$ (II) signal as the acid concentration increased (Figure 6), and confirms the easy elution of copper from these materials. The Dowex material on the other hand does not show such a rapid reduction in capacity with a capacity of $68 \mathrm{mg} / \mathrm{g}$ achieved at $\mathrm{pH}=0.5$, which is still $75 \%$ of the material capacity at $\mathrm{pH}=2$. This result also confirms the earlier EPR observations on the difficulty of removing all the $\mathrm{Cu}(\mathrm{II})$ signal even at high acid concentrations.

\section{CONCLUSIONS}

A combined CW EPR, DFT, and UV-vis study of local metal binding environments in the Dowex and CuWRAM resins for $\mathrm{Cu}$ extraction from aqueous media was undertaken to provide a better understanding of previously reported uptake kinetics and operational properties. Three distinct binding environments (species 2, 3, and A) were identified from the complex EPR spectrum of the "as received" Dowex resin, and a further three environments (species 5, 6, and B) were identified in the CuWRAM case. Comparison to model complexes in solution, containing the "free ligand" species, aided the characterization of two species out of three in each case. An unknown species was observed in each of the chelating exchange resins (species $\mathbf{A}$ and $\mathbf{B}$ ), which were not observed in solution and which were proposed to be based on weakly bound, intermolecular type coordinations to the resin frameworks. The binding behavior of the indicated species was monitored during dehydration studies and elution with $\mathrm{H}_{2} \mathrm{SO}_{4} / \mathrm{NH}_{4} \mathrm{OH}$ to better understand the nature and relative binding affinities of $\mathrm{Cu}$ species adsorbed onto the surface.

The EPR signals associated with unknown species and the heteroleptic complexes (species A and 2, and species B and 6, respectively) were observed to diminish with dehydration, indicating the presence of water within the coordination sphere which may act to "block" donor atoms. Selective unloading studies confirmed that the unknown species, $\mathbf{A}$ and $\mathbf{B}$, are weakly bound and are first to be removed from the resin framework upon elution. A mixture of remaining species is indicated in the intermediate eluent concentration ranges as nonregular line shapes in the parallel hyperfine region of the EPR spectra. The EPR investigations were supported by UVvis measurements to determine equilibrium $\mathrm{Cu}(\mathrm{II})$ capacity versus $\mathrm{pH}$ and estimated equilibrium formation constants for respective uptake mechanisms.

\section{ASSOCIATED CONTENT}

\section{S Supporting Information}

The Supporting Information is available free of charge on the ACS Publications website at DOI: 10.1021/acs.inorgchem.8b01519.

X-band CW EPR spectra of aqueous frozen solutions of $\mathrm{Cu}: \mathrm{PA}$ and $\mathrm{Cu}: \mathrm{BPA}$ complexes with increasing $\mathrm{Cu}$ to ligand ratios; X-band EPR spectra the Cu loaded Dowex sample under hydration/dehydration conditions; Simulation of Dowex and CuWRAM resins with individual components; Peisach-Blumberg plot of $g_{\|} / A_{\|}$parameters for identified species; X-band EPR spectra of Dowex after washing with increasing concentrations of base; UV-vis metal-ligand titration graphs and modeling; Simulated and calculated $g / A$ frames of identified species (PDF)

\section{AUTHOR INFORMATION}

\section{Corresponding Author}

*E-mail: murphydm@cardiff.ac.uk.

ORCID 우

Damien M. Murphy: 0000-0002-5941-4879

\section{Notes}

The authors declare no competing financial interest.

\section{ACKNOWLEDGMENTS}

We would like to thank the EPSRC and Johnson Matthey for funding JS (through an iCASE award). Further support through EP/P019951 is also acknowledged.

\section{REFERENCES}

(1) Glöser, S.; Soulier, M.; Tercero Espinoza, L. A. Dynamic Analysis of Global Copper Flows. Global Stocks, Postconsumer Material Flows, Recycling Indicators, and Uncertainty Evaluation. Environ. Sci. Technol. 2013, 47, 6564-6572.

(2) Wang, L. K., Vaccari, D. A., Li, Y.; Shammas, N. K. Chemical Precipitation. In Physicochemical Treatment Processes; Humana Press: Totowa, NJ, 2005; pp 141-197.

(3) Hsu, Y. J.; Kim, M. J.; Tran, T. Electrochemical study on copper cementation from cyanide liquors using zinc. Electrochim. Acta 1999, $44,1617-1625$.

(4) Ritcey, G. M. Solvent Extraction in Hydrometallurgy: Present and Future. Tsinghua Sci. Technol. 2006, 11, 137-152.

(5) Dorfner, K. Ion Exchangers; Walter de Gruyter \& Co.: Berlin, 1991.

(6) Cavaco, S. A.; Fernandes, S.; Quina, M. M.; Ferreira, L. M. Removal of chromium from electroplating industry effluents by ion exchange resins. J. Hazard. Mater. 2007, 144, 634-638.

(7) Sahni, S. K.; Reedijk, J. Coordination chemistry of chelating resins and ion exchangers. Coord. Chem. Rev. 1984, 59, 1-139.

(8) Arbabi, M.; Golshani, N. Removal of copper ions Cu (II) from industrial wastewater: A review of removal methods. Int. J. Epidemiol. Res. 2016, 3, 283-293.

(9) Gode, F.; Pehlivan, E. A comparative study of two chelating ionexchange resins for the removal of chromium(III) from aqueous solution. J. Hazard. Mater. 2003, 100, 231-243.

(10) Hoek, P. J.; Reedijk, J. Coordination of transition-metal ions by chelating cation-exchange resins; $\mathrm{Co}(\mathrm{II}), \mathrm{Ni}(\mathrm{II})$ and $\mathrm{Cu}(\mathrm{II})$ coordinated by the iminodiacetate group in Chelex-100. J. Inorg. Nucl. Chem. 1979, 41, 401-404.

(11) Chen, T.; et al. Insight into Highly Efficient Coremoval of Copper and p-Nitrophenol by a Newly Synthesized Polyamine Chelating Resin from Aqueous Media: Competition and Enhancement Effect upon Site Recognition. Environ. Sci. Technol. 2013, 47, 13652-13660.

(12) Sirola, K.; Laatikainen, M.; Lahtinen, M.; Paatero, E. Removal of copper and nickel from concentrated $\mathrm{ZnSO}_{4}$ solutions with silicasupported chelating adsorbents. Sep. Purif. Technol. 2008, 64, 88-100.

(13) Abd-El-Aziz, A. S.; Carraher, C. E., Jr; Pittman, C. U., Jr; Zelden, M. Macromolecules Containing Metal and Metal-Like Elements; John Wiley \& Sons: Hoboken, NJ, 2005; Vol. 4.

(14) Kołodyńska, D.; Sofińska-Chmiel, W.; Mendyk, E.; Hubicki, Z. DOWEX M 4195 and LEWATIT MonoPlus TP 220 in Heavy Metal Ions Removal from Acidic Streams. Sep. Sci. Technol. 2014, 49, 20032015. 
(15) Sirola, K.; Laatikainen, M.; Paatero, E. Effect of temperature on sorption of metals by silica-supported 2-(aminomethyl)pyridine. Part II: Sorption dynamics. React. Funct. Polym. 2010, 70, 56-62.

(16) Sirola, K.; Laatikainen, M.; Paatero, E. Effect of temperature on sorption of metals by silica-supported 2-(aminomethyl)pyridine. Part I: Binding equilibria. React. Funct. Polym. 2010, 70, 48-55.

(17) Healy, M. R.; Carter, E.; Fallis, I. A.; Forgan, R. S.; Gordon, R. J.; Kamenetzky, E.; Love, J. B.; Morrison, C. A.; Murphy, D. M.; Tasker, P. A. EPR/ENDOR and Computational Study of Outer Sphere Interactions in Copper Complexes of Phenolic Oximes. Inorg. Chem. 2015, 54, 8465-8473.

(18) Stoll, S.; Schweiger, A. Easyspin, a comprehensive software package for spectral simulation and analysis in EPR. J. Magn. Reson. 2006, 178 (1), 42-55.

(19) Schmidt, M. W.; Baldridge, K. K.; Boatz, J. A.; Elbert, S. T.; Gordon, M. S.; Jensen, J. H.; Koseki, S.; Matsunaga, N.; Nguyen, K. A.; Su, S.; Windus, T. L.; Dupuis, M.; Montgomery, J. A., Jr. General atomic and molecular electronic structure system. J. Comput. Chem. 1993, 14, 1347-1363.

(20) Becke, A. D. Density-functional thermochemistry. III. The role of exact exchange. J. Chem. Phys. 1993, 98, 5648-5652.

(21) Lee, C.; Yang, W.; Parr, R. G. Development of the ColleSalvetti correlation-energy formula into a functional of the electron density. Phys. Rev. B: Condens. Matter Mater. Phys. 1988, 37, 785-789.

(22) Vosko, S. H.; Wilk, L.; Nusair, M. Accurate spin-dependent electron liquid correlation energies for local spin density calculations: a critical analysis. Can. J. Phys. 1980, 58, 1200-1211.

(23) Stephens, P. J.; Devlin, F. J.; Chabalowski, C. F.; Frisch, M. J. $\mathrm{Ab}$ Initio Calculation of Vibrational Absorption and Circular Dichroism Spectra Using Density Functional Force Fields. J. Phys. Chem. 1994, 98, 11623-11627.

(24) Grimme, S.; Antony, J.; Ehrlich, S.; Krieg, H. A consistent and accurate $\mathrm{ab}$ initio parametrization of density functional dispersion correction (DFT-D) for the 94 elements H-Pu. J. Chem. Phys. 2010, $132,154104$.

(25) Weigend, F.; Ahlrichs, R. Balanced basis sets of split valence, triple zeta valence and quadruple zeta valence quality for $\mathrm{H}$ to $\mathrm{Rn}$ : Design and assessment of accuracy. Phys. Chem. Chem. Phys. 2005, 7, 3297-3305.

(26) Marenich, A. V.; Cramer, C. J.; Truhlar, D. G. Universal Solvation Model Based on Solute Electron Density and on a Continuum Model of the Solvent Defined by the Bulk Dielectric Constant and Atomic Surface Tensions. J. Phys. Chem. B 2009, 113, 6378-6396.

(27) Kozuch, S.; Martin, J. M. L. Spin-component-scaled double hybrids: An extensive search for the best fifth-rung functionals blending DFT and perturbation theory. J. Comput. Chem. 2013, 34, 2327-2344.

(28) Kozuch, S.; Martin, J. M. L. DSD-PBEP86: in search of the best double-hybrid DFT with spin-component scaled MP2 and dispersion corrections. Phys. Chem. Chem. Phys. 2011, 13, 20104-20107.

(29) Neese, F. The ORCA program system. Wiley Interdiscip. Rev. Comput. Mol. Sci. 2012, 2, 73-78.

(30) Neese, F.; Wennmohs, F.; Hansen, A.; Becker, U. Efficient, approximate and parallel Hartree-Fock and hybrid DFT calculations. A 'chain-of-spheres' algorithm for the Hartree-Fock exchange. Chem. Phys. 2009, 356, 98-109.

(31) Hellweg, A.; Hättig, C.; Höfener, S.; Klopper, W. Optimized accurate auxiliary basis sets for RI-MP2 and RI-CC2 calculations for the atoms $\mathrm{Rb}$ to $\mathrm{Rn}$. Theor. Chem. Acc. 2007, 117, 587-597.

(32) Weigend, F. Accurate Coulomb-fitting basis sets for $\mathrm{H}$ to Rn. Phys. Chem. Chem. Phys. 2006, 8, 1057-1065.

(33) O’Boyle, N. M.; Banck, M.; James, C. A.; Morley, C.; Vandermeersch, T.; Hutchison, G. R. Open Babel: An open chemical toolbox. J. Cheminf. 2011, 3, 33.

(34) Halgren, T. Merck molecular force field. I. Basis, form, scope, parameterization, and performance of MMFF94. J. Comput. Chem. 1996, 17, 490-519.
(35) Halgren, T. Merck molecular force field. II. MMFF94 van der Waals and electrostatic parameters for intermolecular interactions. J. Comput. Chem. 1996, 17, 520-552.

(36) Halgren, T. Merck molecular force field. III. Molecular geometries and vibrational frequencies for MMFF94. J. Comput. Chem. 1996, 17, 553-586.

(37) Halgren, T. Merck molecular force field. V. Extension of MMFF94 using experimental data, additional computational data, and empirical rules. J. Comput. Chem. 1996, 17, 616-641.

(38) Halgren, T.; Nachbar, R. Merck molecular force field. IV. Conformational energies and geometries for MMFF94. J. Comput. Chem. 1996, 17, 587-615.

(39) Adamo, C.; Barone, V. Toward reliable density functional methods without adjustable parameters: The PBE0 model. J. Chem. Phys. 1999, 110, 6158-6170.

(40) Chong, D. P. Recent advances in density functional methods; World Scientific: Singapore, 1995.

(41) Hedegård, E. D.; Kongsted, J.; Sauer, S. P. A. Improving the calculation of Electron Paramagnetic Resonance hyperfine coupling tensors for d-block metals. Phys. Chem. Chem. Phys. 2012, 14, 1066910676.

(42) Neese, F. Efficient and accurate approximations to the molecular spin-orbit coupling operator and their use in molecular gtensor calculations. J. Chem. Phys. 2005, 122, 034107.

(43) Gao, J.; Liu, F.; LIng, P.; Lei, J.; Li, L.; Li, C.; Li, A. High efficient removal of $\mathrm{Cu}(\mathrm{II})$ by a chelating resin from strong acidic solutions: Complex formation and DFT certification. Chem. Eng. J. 2013, 222, 240-247.

(44) Peisach, J.; Blumberg, W. E. Structural implications derived from the analysis of electron paramagnetic resonance spectra of natural and artificial copper proteins. Arch. Biochem. Biophys. 1974, $165,691-708$.

(45) Froncisz, W.; Hyde, J. S. Broadening by strains of lines in the $g$ parallel region of $\mathrm{Cu}^{2+}$ EPR spectra. J. Chem. Phys. 1980, 73, 31233131.

(46) Tabbì, G.; Giuffrida, A.; Bonomo, R. P. Determination of formal redox potentials in aqueous solution of copper(II) complexes with ligands having nitrogen and oxygen donor atoms and comparison with their EPR and UV-vis spectral features. J. Inorg. Biochem. 2013, $128,137-145$.

(47) Palaniandavar, M.; Butcher, R. J.; Addison, A. W. Dipicolylamine Complexes of Copper(II): Two Different Coordination Geometries in the Same Unit Cell of $\mathrm{Cu}(\text { Dipica })_{2}\left(\mathrm{BF}_{4}\right)_{2}$. Inorg. Chem. 1996, 35, 467-471.

(48) Niven, M. L.; Percy, G. C.; Thornton, D. A. The infrared spectra of 2-aminomethylpyridine complexes of metal(II) ions. J. Mol. Struct. 1980, 68, 73-80. 